very slow atomic motion and to detect coherent phonons.

In the papers on liquids and gases the main concern is with the effects on the nuclear resonance of fluctuations due to motion in inter- and intra-molecular interactions. These papers and those on dielectric relaxation are not without interest, but they are really too fow and heterogeneous to be representative of their fields, and would have conveyed their benefits more widely in the published literature than at a conference devoted primarily to nuclear resonance in solids.

While the general reader must not expect a coherent picture of nuclear magnetic rosonance in solids, he will find a wide sample of activities in the field. For such a reader and for the student entering research, the compre. hensive range of topics and associated references will be a useful entry into the specialist literature. The specialist himself will be conscious of the delay in publication but should find the documontation and the full edited discussions useful. The production of the book is excellent, with clear diagrams and a well edited text containing few mistalies.

J. BUTTERWORTH

\section{SELENIUM, TELLURIUM AND POLONIUM}

The Chemistry of Selenium, Tellurium and Polonium By K. W. Bagnall. (Topics in Inorganic and General Chemistry, Monograph 7.) Pp. viii + 200. (Amsterdam, London and New York: Elsevier Publishing Company, 1966.) $70 s$.

DR. BAgNall's book concerns three somewhat neglected members of the periodic table. The need for a manageable, concise and up-to-date treatment of selenium, tellurium and polonium has been folt by undergraduates and research students alike, and in the rather over. populated world of chemical 'literature' it is pleasant to welcome just such a book. One theme of the book is to accommodate polonium in the context of the chemistry of the heavier elements of Group VI $B$, but knowledge of polonium is still unfortunately such that comparisons are inevitably limited. The attempt to redress the balance of knowledge has meant that certain aspects of the chemistry of tellurium and more particularly of selenium have not received due attention. To undergraduates the volume will present no problems, being set out in the classical format evident from the following chapter headings: "The Discovery and Uses of the Elements"; "Separation, Purification and Determination"; "The Elements, the Hydrides and other Derivatives"; "The Oxides, Oxo-acids, Sulphides and Sulphur-oxo-acids"; "Halides"; "Salts formed with Oxo-acids"; and "Carbon Compounds". Factual information is well provided, and some of the anomalies and uncertainties which have beon underlined should certainly stimulato the interest anticipated by the author. Explanations come less easily, admittedly, and a judicious sentence or two would have helped to outline the shape of the wood as well as the trees; for example, the stereochemistry of selenium (IV) and tellurium (IV) compounds is described only for individual molecules despite the fact that the general stereochemical features for such systems are now fairly well defined.

Readers will appreciate the lucid style of the book, which is pleasingly free of ambiguity; the fow errors, such as the reference to the infra-red frequencies $v_{1}-v_{6}$ of $\mathrm{SeF}_{6}$, and a rather misleading description of the $\mathrm{SeF}_{4}$ molecule as a "distorted tetrahedron", do not alter the general impression. For the research worker there is a useful bibliography. This has been pruned to restrict it to reasonable proportions, and the list of references is by no means comprehensive enough to make this the com- plete source-book of information about selenium and tellurium. Despite the obvious need to limit the number of references, it is questionable whether the signposts to the appropriate literature are always as numerous and well chosen as they might be.

Except for a few trivial errors, the printing and general presentation of the volume leave nothing to be desired. This is a sound and useful account that will be of value to students at all levels, and to specialist chemists as a first reference in matters concerning selenium, tellurium and polonium.

A. J. Downs

\section{THE SOLAR CORONA}

\section{Physics of the Solar Corona}

By I. S. Shklovskii. Second edition. Translated by L. A. Fenn. Translation edited by A. Beer. Additional material translated and edited by A. J. Meadows. (International Series of Monographs on Natural Philosophy, Vol. 6.) Pp. $\mathrm{x}+475$. (London and New York: Pergamon Press, Ltd., 1965.) 120s. net.

Solar physics has developed within the past decade to an astonishing degree. There are now whole text-books dealing only with some special aspects of solar research. Shklovskii's book, now in its second edition, consists of not fewer than 475 pages of a morphology devoted entirely to present knowledge of the solar corona, a field of research to which the author himself has most actively contributed. The corona is at present of special interest since we are dealing with the outermost atmosphere of the Sun, a subject still presenting many most difficult and unsolved physical problems. Since the outer parts of the solar corona continuously merge into the interstellar space, this whole subject is at the same time of tremendous actual interest for modern space research. These considerations have even given rise to quite a new research line in astrophysics which may rightly be called experimental astrophysics.

The book gives a good survey of present knowledge of the solar corona, including first the more elementary fields of research in this line during the past decade. But then it turns with special emphasis to the most modern investigations and to the connexion with radio astronomy and with research into the interplanetary medium.

The first hundred pages of the book cover the main results of various observations of the corona; a great many of them were collected by solar eclipse observations of various kinds. A further chapter is devoted to the emission and absorption spectra of the corona, a most fascinating field for spectroscopists, followed by a well written review of quantitative chemical analysis of the corona using all the information presented by the available spectroscopic evidence. The author then turns to the physically quite difficult theory for explaining the deviation from thermodynamic equilibrium in the upper layers of the solar atmosphere and in the corona, and he then describes the ultra-violet and X-ray radiation of the Sun, originating mainly within the corona. This chapter also takes into account the influence of the short wave solar radiation on the ionization of the atmosphere of the Earth-important for modern telecommunication. Spectacular results have recently been produced in this line by extended programmes of rocket research. There follows a short outline of modern radio astronomy and of radio emission from the Sun, most of which originates in the highest layers of the Sun and in the corona. The different theories of the solar corona, all still more or less in dispute, are outlined in a further chapter and, finally, there is a discussion of the interplanetary matter between the Sun and the immediate surroundings of the Earth which nowadays is of great importance. 